Archives

\title{
Équivoque épistolaire : un usage de l'exégèse à la cour de France
}

Alain Brunn

\section{(2) OpenEdition}

Journals

Édition électronique

URL : http://journals.openedition.org/ccrh/233

DOI : $10.4000 /$ ccrh.233

ISSN : $1760-7906$

Éditeur

Centre de recherches historiques - EHESS

Édition imprimée

Date de publication : 1 avril 2004

ISSN : 0990-9141

Référence électronique

Alain Brunn, «Équivoque épistolaire : un usage de l'exégèse à la cour de France », Les Cahiers du Centre de Recherches Historiques [En ligne], 33 | 2004, mis en ligne le 05 septembre 2008, consulté le 10 décembre 2020. URL : http://journals.openedition.org/ccrh/233; DOI : https://doi.org/10.4000/ccrh. 233

Ce document a été généré automatiquement le 10 décembre 2020.

Article L.111-1 du Code de la propriété intellectuelle. 


\title{
Équivoque épistolaire : un usage de l'exégèse à la cour de France
}

\author{
Alain Brunn
}

La fortune, puissance de l'événement n'est donc pas un concept, une notion théorique, un principe d'explication. Principe dont la position épistémologique est de disqualifier toute explication, le terme nomme plus précisément

l'impossibilité d'une science de l'action politiquement opportune. Au niveau de l'action, il signifie qu'il n'existe pas de règle capable de faire reconnaître l'action nécessaire. La fortune, l'événement dans son apparition, n'est pas porteur

d'un déterminisme politique sur lequel il serait possible de s'appuyer pour agir, mais d'un possible à saisir ici maintenant par une volonté, une virtù, pour agir de façon cohérente. La fortune nomme dans l'événement la puissance du neutre. Louis Marin, Le Récit est un piège.

1 C'est par cette analyse que Louis Marin rend compte des «bagatelles pour meurtres » disposées par le cardinal de Retz dans ses Mémoires ${ }^{1}$. Il faut prendre la mesure d'une telle proposition. Loin de mettre en place une histoire univoque, toute entière soumise au joug de la providence divine, le politique qu'est Retz installe sur la scène de son texte - et comme la leçon de celui-ci-la «puissance du neutre ", c'est-à-dire l'indifférence profonde de ce qui arrive à ses suites, la plasticité de l'événement qui n'est jamais déterminé en soi, mais toujours ouvert à des usages contradictoires. Le neutre prend alors le visage de l'équivoque, de ce qui se donne librement, dans une pleine, entière et contradictoire disponibilité. 
2 Sur les raisons d'une telle lecture de l'histoire chez de nombreux mémorialistes de l'âge classique, il faudrait sans doute s'interroger, et souligner les raisons théologico-politiques de cette neutralisation de l'événement, l'inscrire dans les pratiques d'un ordre en crise frappé en plein cœur par la Fronde, et le lier - sans doute pour un Retz, assurément chez un La Rochefoucauld - aux mutations du romanesque au XVII ${ }^{\mathrm{e}}$ siècle. Tel ne sera pas notre objet: plus modestement, nous voudrions rapprocher, à travers l'étude d'un cas particulier, cette conception équivoque de l'histoire de certains objets présents tout au long de l'âge classique, que l'on a pu dire fragmentaires ou discontinus, lettres, énigmes, maximes ${ }^{2}$; objets surtout, voudrions-nous montrer, caractérisés par une disponibilité particulière à l'équivoque en raison de leur fragilité contextuelle : sans contexte fort pour décider de leur sens - et leur caractéristique est bien de ne s'inscrire que secondairement dans une série, puisqu'ils peuvent fonctionner sans elle -, ils sont ouverts à toutes les nominations possibles, à toutes les décisions herméneutiques envisageables, incarnant ainsi avec force l'inquiétante "puissance du neutre». Il sera donc ici question de l'équivoque envisagée comme fabrication et non comme fait : l'équivoque comme usages (contradictoires) du neutre, donc.

Quel objet plus anonyme, dans cette perspective, qu'une lettre sans auteur et sans destinataire? Quel objet plus neutre qu'une lettre perdue? De ces lettres perdues, le grand siècle a laissé de nombreux exemples, de l'Histoire amoureuse des Gaules à La Princesse de Clèves. Parmi tous ceux-ci, une anecdote a eu les honneurs de nombreux mémoires contemporains, une histoire de lettre perdue advenue en 1643 à la cour de France. Anecdote toute entière construite autour d'un objet équivoque: elle repose sur le maniement, par les différents protagonistes, d'une situation réversible, d'un événement neutre; objet équivoque, donc, qui conduit sur une intrigue équivoque, c'est-à-dire sur une intrigue «neutre ", réversible, mais aussi anecdote elle-même équivoque, parce que les traces que nous en avons gardées (les différents textes qui relatent cet épisode) sont eux-mêmes ambivalents.

Le sujet de cette anecdote est ainsi éminemment romanesque, matière de fiction; en témoigne le Dictionnaire de Furetière :

ÉQUIVOQUE, est quelquefois une beveuë, une inadvertance qui nous fait prendre une chose pour une autre. Plusieurs intrigues de Romans sont fondées sur des équivoques de billets rendus à ceux à qui ils ne s'adressaient pas.

Mais une telle «intrigue de roman» a eu en effet lieu à la cour en 1643 ; la fiction a rencontré la référence : au risque d'ajouter à l'équivoque, on en présentera ici un rapide résumé.

5 En 1643, Richelieu et Louis XIII sont morts; le pouvoir est l'objet de luttes à la cour de France, et deux clans particulièrement rivalisent pour conduire la régente Anne d'Autriche à leur donner l'avantage, les Vendôme d'une part, les Condé d'autre part ; ces deux maisons sont de sang royal, et ont les mêmes ambitions. Mazarin, dont l'autorité était mal établie, ne pouvait que s'appuyer sur l'une pour abaisser les prétentions d'une autre; les déséquilibres ne s'en poursuivaient pas moins. La lettre perdue est un des épisodes de cet affrontement. Dans ce cadre prennent place les protagonistes du drame: Madame de Montbazon était la maitresse de M. de Longueville, qui l'avait quittée pour épouser Anne-Geneviève de Bourbon-Condé, sœur du futur Grand Condé; elle l'avait remplacé par Beaufort, l'un des Vendômes, et autour d'eux s'était constituée la cabale des Importants; Madame de Longueville, quant à elle, "aimait véritablement " Coligny (l'affirmation est de Retz). C'est dans cette situation de double rivalité - politique et 
amoureuse-que deux lettres, trouvées par Madame de Montbazon, sont par elle attribuées à une Madame de Longueville s'adressant à Coligny. En fait, elles avaient sans doute été écrites par Madame De Fouquerolles au marquis de Maulévrier. Quoi qu'il en soit, l'anecdote se répand, fait scandale ; les Condé, par la voix de la princesse de Condé (la mère de Madame de Longueville) demandent réparation à la Reine, et l'obtiennent, sous la forme d'un démenti public apporté par Madame de Montbazon dans le salon de la princesse de Condé. L'affaire aura des suites plus graves, mais qui ne nous intéresseront pas parce qu'elles débordent notre cadre : après un nouvel affrontement chez Regnard de Madame de Montbazon et de Madame la princesse, Beaufort sera disgracié ; un an plus tard, en 1644, sur la place Royale, un duel de Guise (pour Madame de Montbazon) et de Coligny (pour Madame de Longueville) conduira à la mort du second.

6 Après ce résumé de mauvaise méthode, il faut indiquer les traces de cette équivoque. L'affaire de la lettre perdue n'est en effet aujourd'hui plus qu'une série de textes: il y a plusieurs extraits des Mémoires de l'époque qui rapportent l'anecdote, ceux de La Rochefoucauld, qui commence à l'époque à se rapprocher du clan condéen (mais qui s'en éloigne avec la rédaction de ses mémoires), ceux de Madame de Motteville, confidente de la Reine, ceux de Mademoiselle de Montpensier, relativement indépendante (elle est la fille de Gaston d'Orléans, donc plus importante que les clans en présence), ceux enfin, anonymes, cités par Chéruel, particulièrement romanesques (leur anonymat n'est pas pour rien dans ce romanesque) ${ }^{3}$. Et, à l'horizon de la série - mais écartée ici -, il est bien difficile d'oublier une autre histoire de lettre perdue, une "intrigue de roman » qui se souvient sans doute de ces récits, même si elle est toute différente par l'époque et le genre où elle prend place : celle contée par Madame de Lafayette à propos du Vidame de Chartres.

7 Nous lirons donc ces mémoires en perspective pour en dégager les équivoques, non pour savoir ce qui s'est passé ce jour de 1643, mais parce que l'attention portée à une telle anecdote par ses contemporains suscite la curiosité : ce qu'ils y voient, c'est bien autre chose qu'une rivalité politique et galante; c'est un événement central dans l'histoire du siècle, et pour certains une origine de la Fronde. Qu'est-ce alors qui se manifeste dans cette histoire pour qu'elle puisse ainsi se constituer en pivot? Ultime équivoque de la série : celle qui oppose la lecture contemporaine à notre propre lecture, et qu'il faudra bien résoudre (c'est sans doute la seule, d'ailleurs, dont la résolution s'impose).

Car tous ces textes participent d'une même histoire, celle des usages de l'équivoque comme technique de pouvoir. Cette anecdote équivoque, qui est aussi histoire de publication, puisque il y a un lien, apparemment, entre l'équivoque et le privé, entre l'univoque et le public, a en effet pour conséquence de mettre en valeur le rapport de l'équivoque et du pouvoir : l'équivoque est un moyen (toujours fragile et réversible) de s'emparer du neutre de l'événement ; l'équivoque est le visage du neutre. À ce titre, il est la mesure du pouvoir; ce sont en effet des procédures de pouvoir qui viennent caractériser le masque neutre de l'équivoque, procédures de pouvoir qui sont aussi des procédures de savoir, et qui seules peuvent abolir l'équivoque : la fixer en un (définitif ?) univoque. C'est donc bien la question de l'usage de l'équivoque qui est au cœur de cette anecdote : qu'est-ce qui se donne du pouvoir dans l'équivoque? soit, inséparablement (peut-être est-ce la même question) qu'est-ce qui se donne à voir du pouvoir dans l'équivoque? L'équivoque, ainsi, apparaîtrait comme lieu de représentation du pouvoir, lieu privilégié, parce qu'elle n'est pas le lieu propre du pouvoir, mais un lieu qui le convoque comme son terme. 


\section{Le récit de La Rochefoucauld : équivoque et pouvoir}

Le récit que laisse le duc de La Rochefoucauld s'inscrit dans une description romanesque du contexte politique de l'époque: il fait de l'anecdote un moment décisif des affrontements claniques à la cour, et notamment de la rivalité des Importants (une cabale "de la plupart de ceux qui avaient été attachés à la Reine », mais dont La Rochefoucauld ne fait pas partie).

Les choses étaient en ces termes, et le cardinal Mazarin, d'une part, et Mme de Chevreuse et le duc de Beaufort, de l'autre, songeaient avec beaucoup d'application à se détruire. La bonne fortune du Cardinal et l'imprudence du duc de Beaufort et de Mme de Montbazon, dont il était amoureux, fournirent bientôt une occasion, dont le Cardinal sut profiter pour venir à bout de son dessein.

Car l'anecdote est bien une « occasion » à saisir ; comme chez le Retz de Marin, la fortune n'est jamais donnée : elle est à prendre, à fabriquer.

Un jour que Mme de Montbazon gardait la chambre, et que beaucoup de personnes de qualité l'allèrent voir, dont Coligny était du nombre, quelqu'un, sans y penser, laissa tomber deux lettres bien écrites, passionnées, et d'un beau caractère de femme.

C'est avec ce «beau caractère de femme » que naît l'équivoque. La lettre, sans nom, est sans contexte, mais elle en dessine un, même flou, que tout lecteur est appelé à finir : elle peut avoir plusieurs auteurs; plus encore, elle n'est rien en elle-même; elle n'est qu' occasion (le terme revient), chance à saisir :

Mme de Montbazon, qui haïssait Mme de Longueville, se servit de cette occasion pour lui faire une méchanceté. Elle crut que le style et l'écriture pourraient convenir à Mme de Longueville, bien qu'il y eût peu de rapport et qu'elle n'y eût aucune part.

Le caractère est donc glosé en style et écriture. Ce à quoi se livre (de façon sauvage) Madame de Montbazon, c'est à une procédure d'attribution d'un texte à un auteur, donc à un travail exégétique, à un travail philologique (comme le Jérôme analysé par Foucault ${ }^{4}$, elle évalue le champ de cohérence théorique et l'unité stylistique du document pour lui attribuer un auteur). Autrement dit, Madame de Montbazon fait de la supposition d'auteur, au sens technique du mot (le terme se trouve d'ailleurs dans le texte des Mémoires). Elle fait œuvre d'exégète et attribue un texte en s'appuyant sur des critères formels complémentaires (style et écriture): elle crée exégétiquement un caractère d'auteur, compatible avec une personne que politiquement elle veut perdre. Aussi, avec le recours au critère du caractère de femme, elle va plus loin qu'une simple opération exégétique ; elle étend en effet ses critères à une étude de l'éthos de l'auteur des lettres.

11 Le texte vient supporter la phrase de l'exégète, et il faudra la rupture du bien que pour que se fasse clairement entendre une autre voix, celle de l'omniscient La Rochefoucauld. Le propos exégétique de Madame de Montbazon n'est pas contredit par une contre analyse, mais sur une simple affirmation du mémorialiste, autrement dit par le seul éthos de La Rochefoucauld. Aussi l'équivocité de la lettre de femme subsiste; mais elle est progressivement surmontée, parce que le texte va progressivement renforcer l'ethos du mémorialiste. L'équivoque sert donc paradoxalement à renforcer l'ethos de l'écrivain, puisque elle permet de le mesurer (d'en donner la mesure); avec elle, c'est donc une question de pouvoir qui est posée. La construction de l'ethos de l'écrivain se fait en effet progressivement, par une minoration de celui de l'exégétique duchesse. 
Elle prévint le duc de Beaufort, pour le faire entrer dans ses sentiments; et tous deux, de concert, firent dessein de répandre dans le monde que Coligny avait perdu des lettres de Mme de Longueville qui prouvaient leur intelligence. Mme de Montbazon me conta cette histoire devant que le bruit en fût répandu : j'en vis d'abord toutes les conséquences, et quel usage le cardinal Mazarin en pourrait faire contre le duc de Beaufort et contre tous ses amis. J'avais peu d'habitude alors avec Mme de Longueville ; mais j'étais particulièrement serviteur de M. le duc d'Enghien et ami de Coligny. pouvoir. Or ce pouvoir de l'équivoque n'est tel que d'être utilisé dans le public: à l'inverse, c'est «son particulier » qui conduit La Rochefoucauld à intervenir. Il voit bien en effet que l'objet équivoque articule particulier et politique, et que c'est le public qui va être touché par l'équivoque d'un objet privé en fait destiné à jouer un rôle dans l'affrontement de Mazarin et des Importants. La lettre équivoque, que Madame de Montbazon a tenté d'univoquer pour la faire servir à son dessein, ne perd pas son principal caractère : le cardinal, lui aussi, peut en faire usage.

L'objet (la lettre perdue) est donc bien un objet équivoque : la lettre sans auteur, et sans destinataire, a du fait même plusieurs sens possibles; univoquée d'abord par Madame de Montbazon, qui l'attribue à Madame de Longueville, elle peut être contre univoquée, et ainsi servir à Mazarin; tout se passe comme si les opérations exégétiques menées par Madame de Montbazon n'affectaient pas vraiment la nature de l'objet sur lequel elles portent; la lettre équivoque ne s'univoque qu'au prix d'une double opération, philologique et politique, dont la seconde partie seule semble décisive. Mazarin peut en effet retourner l'univocité de la lettre contre Montbazon, donc recourir (après même l'univocation) à son premier caractère équivoque.

Je connaissais la malignité du duc de Beaufort et de Mme de Montbazon, et je ne doutai point que ce ne fût une méchanceté qu'ils voulaient faire à Mme de Longueville. Je fis tous mes efforts pour engager Mme de Montbazon, par la crainte des suites, à brûler les lettres devant moi, et à n'en parler jamais : elle me l'avait promis, mais le duc de Beaufort la fit changer.

Comment alors sortir de l'équivoque ? Comment mettre un terme au pouvoir destructeur de l'objet équivoque ? La solution que propose La Rochefoucauld est radicale : il s'agit de brûler la lettre. Pour sortir de l'équivoque, il faut détruire l'objet équivoque. Mais Beaufort, fidèle à sa catastrophique réputation, intervient. Se précise alors, en même temps que le pouvoir de l'équivoque (et derrière lui), la relation du pouvoir à l'équivoque.

[Mme de Montbazon] se repentit bientôt de n'avoir pas suivi mon conseil : cette affaire devint publique, et toute la maison de Condé s'y intéressa comme elle devait. Cependant celui qui avait véritablement perdu les lettres était de mes amis, et il aimait la personne qui les avait écrites. Il voyait que les lettres seraient indubitablement reconnues, puisque Monsieur le Prince, Madame la Princesse et Mme de Longueville voulaient les montrer publiquement pour convaincre Mme de Montbazon d'une noire supposition, par la différence de l'écriture. Dans cet embarras, celui qui avait perdu les lettres souffrit tout ce qu'un honnête homme doit souffrir dans une telle rencontre : il me parla de sa douleur, et me pria de tenter toutes choses pour le tirer de l'extrémité où il se trouvait.

L'affaire devient donc publique, de particulière qu'elle était : nature de l'équivoque, qui articule secret et dévoilement; travail de l'exégèse, qui met à l'épreuve de la contradiction ouverte sa recherche discrète. Mais, sortant ainsi du privé, l'équivoque change de valeur, qui devient incontrôlable par ses premiers univocateurs (Madame de Montbazon et Beaufort). Car la publicité voulue par les Condé a pour effet de défaire la 
première univocation de la lettre: l'équivocité particulière, voulue par Madame de Montbazon, lui permettait d'univoquer la lettre dans son particulier - de faire de son particulier le lieu, et le critère, de l'interprétation; c'est seulement dans un second temps que cet univoque se voyait publié. Mais, avec le passage au public, la lettre devient incontrôlable pour le particulier de la duchesse : s'il y a passage de la lettre au public, la lettre redevient équivoque, et donc elle est susceptible d'être contre univoquée, c'est-àdire imputée à quelqu'un d'autre que Madame de Longueville.

Mais ce public n'est pas non plus sans danger; l'intervention d'un témoin privilégié, l'auteur véritable (et anonyme, ce qui l'atteste paradoxalement en maintenant la lettre dans l'équivoque), vient dire le danger de la publication : là encore, l'objet équivoque se voit caractérisé comme dangereux au moment même où son caractère équivoque doit pouvoir disparaître (au passage, c'est aussi l'ethos de La Rochefoucauld qui se voit encore renforcé). À cause de cet auteur, on ne peut sortir de l'univocité créée par Madame de Montbazon par une publication de l'univoque contraire (la vérité) : les lettres ne doivent en effet pas être publiquement reconnues, sous peine de causer une nouvelle catastrophe - une autre issue funeste, que l'intervention du narrateur va permettre d'éviter.

Je le servis heureusement; je portai les lettres à la Reine, à Monsieur le Prince et à Madame la Princesse; je les fis voir à Mme de Rambouillet, à Mme de Sablé et à quelques amies particulières de Mme de Longueville; et aussitôt que la vérité fut pleinement connue, je les brûlai devant la Reine, et délivrai par là d'une mortelle inquiétude les deux personnes intéressées. Bien que Mme de Longueville fût entièrement justifiée dans le monde, Mme de Montbazon ne lui avait point encore fait les réparations publiques qu'elle lui devait : les conditions en furent longtemps disputées, et tous ces retardements augmentaient l'aigreur.

17 Si l'on sort du public, c'est donc à condition d'entrer dans un autre particulier que celui de Madame de Montbazon, un particulier plus fort parce que la reine s'y joint. Autrement dit, c'est une publication auprès du pouvoir qui va finalement décider du sort de l'objet équivoque : procédure exégétique nouvelle et définitive, qui s'autorise du pouvoir pour fixer cette univocation, pour fixer l'objet équivoque dans un sens univoque. Les lettres brûlées viennent alors signifier que l'équivoque est devenu univoque. Mais le critère décisif n'est pas celui de la vérité : il est celui du pouvoir. L'exégèse est affaire de décision, et à ce titre dépend des rapports de force, comme va venir l'expliciter la suite du récit.

Le duc d'Enghien venait de prendre Thionville; il était prêt de finir la campagne, et il revenait outré de colère et d'indignation de l'injure que Madame sa sœur avait reçue. La crainte de son ressentiment, plus que toute autre raison, fit soumettre Mme de Montbazon à tout ce qu'on lui voulut imposer. Elle alla, à une heure marquée, à l'hôtel de Condé trouver Madame la Princesse, qui n'avait pas voulu que Mme de Longueville y fût présente ; toutes les personnes de la plus grande qualité s'y étaient rendues, pour être témoins d'un discours qu'on avait prescrit à Mme de Montbazon, et qu'elle fit pour excuser sa faute et en demander pardon.

L'avantage est pris par les Condé, en raison de la gloire et du pouvoir que sa victoire militaire confère à Condé. Mais, loin d'en rester à une univoque privée (la preuve faite devant la reine), le récit évoque une univoque que les excuses de Madame de Montbazon rendent publique. Rien ne montre alors avec plus de force combien c'est bien le pouvoir qui décide, plus qu'aucune autre chose, de la vraie interprétation, que l'exégèse - et partant la vérité - est affaire de puissance. À l'équivoque privée de la lettre perdue, sans auteur ni destinataire, venait répondre l'univoque privée de l'exégèse calomnieuse ; à l'univoque publique de l'attribution et de la rumeur vient répondre une nouvelle 
équivoque privée (la lettre n'est pas de Madame de Longueville - il n'est pas d'autre détermination que négative de l'auteur de la lettre, ce que vient manifester la destruction de l'objet équivoque: la contre univocation est aussi une rééquivocation ${ }^{5}$ ), que publie l'univoque des excuses de Madame de Montbazon.

Le neutre faisait apparaître la laïcité de la fortune, la providence déchue ; l'équivoque fait apparaître l'indifférence de la vérité, la raison politique de l'exégèse.

\section{Le récit de Madame de Motteville : équivoque et passions}

Mais si l'exégèse est question de pouvoir, si l'équivoque convoque toujours le pouvoir comme son terme, quel récit se donne le pouvoir de l'équivoque ? S'il n'est pas de récit laissé par la reine, il en est un qu'a laissé Madame de Motteville, sa confidente, exemplaire à bien des égards parce que le pouvoir de la Providence y est réhabilité.

Il arriva sur ce temps là une aventure qui démêla toutes les intrigues de la cour, et qui fut cause que $\mathrm{M}$. le cardinal se vit, bientôt après, parfaitement établi dans l'élévation et la puissance qu'il désirait d'avoir. Ce fut une providence de Dieu toute particulière qui fit que les mêmes choses dont les brouillons voulurent se servir pour renverser la cour furent ce qui la régla, aux dépens néanmoins de beaucoup d'honnêtes gens. Mais, de tant d'événements extraordinaires, il s'en faut rapporter à cette cause première qui veut le bien et permet le mal, soit pour notre récompense, soit pour notre punition.

Les dames sont d'ordinaire les premières causes des plus grands renversements des États; et les guerres, qui ruinent les royaumes et les empires, ne procèdent presque jamais que des effets que produisent ou leur beauté ou leur malice. La duchesse de Montbazon, qui a tenu dans notre siècle le premier rang de la beauté et de la galanterie, étant belle-mère de la duchesse de Chevreuse, était aussi bien qu'elle de la cabale des Vendôme, non tant par l'intérêt de sa belle fille que parce que le duc de Beaufort était amoureux d'elle. Par conséquent ces dames étaient opposés à madame la princesse qui n'aimait ni l'une ni l'autre, et qui selon ce que j'ai dit, favorisait M. le cardinal par la haine qu'elle avait contre le garde des sceaux de Châteauneuf.

Outre ces différents intérêts, il y en avait encore un fort grand entre Mme de Longueville, fille de madame la princesse, et la duchesse de Montbazon. Cette belle et jeune demoiselle de Bourbon, forcée par M. le prince son père, avait épousé le duc de Longueville, qui était le plus grand seigneur qu'elle pût épouser, à cause de ses grands biens; et qui suivait immédiatement les princes du sang, et ne pouvait se considérer comme tout à fait digne d'elle, soit à cause de sa naissance, soit à cause de son âge; outre qu'il était amoureux de madame de Montbazon. Ces deux personnes, parmi tant de raisons de ne se pouvoir aimer, avaient de grandes dispositions à se nuire ; et la parfaite beauté de madame de Longueville, sa jeunesse et sa propre grandeur, la conviaient souvent à regarder sa rivale avec mépris.

21 Le récit providentialise ainsi l'intrigue... et l'équivoque : il y a plus, sans doute, que la reconnaissance, dans le lieu du pouvoir terrestre, du pouvoir spirituel. Les intérêts divers sont présentés, mais lus d'abord à travers les maximes galantes et curiales des sexes et des préséances.

C'est dans ce cadre que peut se mener le récit ; on retrouve la plupart des éléments mis en place par La Rochefoucauld que Madame de Motteville a sans doute lu, et notamment la question de l'écriture de femme, sur quoi reposait toute l'équivoque textuelle. Mais le texte 
prend une coloration plus clairement galante, en même temps que plus romanesque (et la providence alors n'y est pas pour rien).

Il arriva donc qu'un jour madame de Montbazon étant chez elle dans un grand cercle, une de ses demoiselles trouva une lettre dans sa chambre, et, l'ayant ramassée, la porta à sa maîtresse. Cette lettre se trouva d'une écriture de femme qui écrivait tendrement à quelqu'un qu'elle ne haïssait pas. Comme pour l'ordinaire de telles matières sont toujours l'entretien de toutes les compagnies, et qu'on préfère celles-là à toute autre chose, on ne négligea pas le sujet de risée que cette lettre donna à ceux qui composaient celle de madame de Montbazon. De la gaieté on vint à la curiosité, de la curiosité au soupçon, et du soupçon on passa jusqu'à décider qu'elle était tombée de la poche de Coligny, qui venait de sortir, et qui, à ce qui se disait à l'oreille, avait de la passion pour madame de Longueville. Cette princesse était alors dans une grande réputation de vertu et de sagesse, mais elle ne laissait pas d'être soupçonnée de ne pas haïr l'adoration et les louanges. C'est l'ordinaire faiblesse de toutes les dames qui, sous des noms de respect et sous les apparences d'une feinte adoration, deviennent elles-mêmes les esclaves de ceux qui font semblant de leur donner de l'encens.

Les personnages présentés, c'est en fait une histoire du passage de l'équivoque à l'univoque qui se donne à lire, sous le signe ambigu des passions : on passe de l'équivoque à l'univoque comme on passe de la risée à la gaieté, puis à la curiosité qui se fait soupçon avant de se révéler décision. Autrement dit, le procès d'attribution est donc fondé sur le pathos, c'est-à-dire - de façon très aristotélicienne - non pas sur l'ethos de l'auteur (la figure de l'exégétique duchesse), mais sur les passions du public: comme la galante de Molière, Madame de Montbazon pourrait bien être défendue par le blâme de son public (avec toute l'ambiguïté du possessif).

La maxime qui finit le passage vient alors justifier l'attribution; et parce qu'il y a attribution, il peut $\mathrm{y}$ avoir publication :

Les premiers qui chez madame de Montbazon dirent après elle que cette lettre était de madame de Longueville ne la crurent pas en effet. Ce ne fut alors qu'une histoire plaisante dont chacun faisait le conte à son ami fort en secret, pour seulement divertir celui qui l'ignorait.

Le rire public continue à présider à l'équivoque. L'histoire plaisante devient conte, l'univocation se fait sous le signe du divertissement: à nouveau, l'univoque se fabrique ; c'était sous le signe de la décision politique chez La Rochefoucauld, c'est sous celui du divertissement chez Madame de Motteville : il n'y a pas forcément si loin de l'un à l'autre . Mais le divertissement est secret, affaire privée, qui se donne à entendre dans le particulier des rieurs. Aussi le récit enchaîne sur cette thématique des passions du public, pour expliquer le « bruit » d'une publication bien différente de celle que faisait apparaître le récit de La Rochefoucauld:

Il ne demeura pas longtemps sans parvenir aux oreilles de madame la princesse [Mme de Condé, mère de Mme de Longueville], qui, selon son naturel altier et vindicatif, le ressentit vivement; et il est presqu'impossible de dire jusques où elle porta sa colère et sa douleur. Madame de Longueville, qui n'était pas moins sensible, mais qui était plus retenue, fut d'avis de n'en pas faire de bruit. La jalousie qu'elle avait contre la duchesse de Montbazon, étant proportionnée à l'amour qu'elle avait pour son mari, ne l'emportait pas si loin qu'elle ne trouvât plus à propos de dissimuler cet outrage ; car il était d'une nature à devoir souhaiter plutôt de l'étouffer que d'en faire une solennelle vengeance.

La publication est donc due à la réaction de la princesse, autrement dit de ce public restreint que déterminait le rire; le divertissement privé à quoi se résumait la 
supposition moqueuse de Madame de Montbazon entraine le grief public de la princesse de Condé: la publication se fait bien en deux temps, par le rire puis par la colère - publication passionnelle, donc, et non point politique comme elle l'était chez La Rochefoucauld; tout se passe comme si (et telle serait la force propre de l'équivoque, lue par Madame de Motteville sous le signe de la divine providence) l'objet épistolaire échappait à tout contrôle parce qu'il déchaîne les passions, parce qu'il était publié par les passions (et par des passions opposées aussi bien). À tout contrôle, donc, mais non à tout usage (et l'on retrouve ici le récit de La Rochefoucauld).

C'est en effet le caractère utilisable de l'équivoque qui guide la réaction de la princesse. Madame de Longueville voulait conserver l'affaire dans le secret des rieurs particuliers; sa mère, pour pouvoir faire un usage politique de l'intrigue, sait qu'il lui faut la changer de contexte, et l'amener dans le public le plus manifeste, celui des « affaires d'état »:

Madame sa mère était animée par beaucoup d'autres grands intérêts : elle savait profiter de l'avantage qu'elle avait d'être entrée dans la maison de Bourbon; et, ne pouvant se retenir, elle fit de cette querelle une affaire d'État. Elle vint trouver la Reine, lui demanda justice, et se plaignit hautement de madame de Montbazon.

La princesse utilise donc l'équivoque pour la contre univoquer (c'est-à-dire en fait la rééquivoquer: n'importe qui a pu écrire cette lettre, sauf sa fille) dans sa publication - affaire d'usage, à nouveau. Mais, si la publication se fait par un recours à l'autorité royale, l'équivoque continue à entraîner un partage passionnel de la Cour :

Voilà toute la cour partagée. Les femmes, qui avaient du respect pour madame la princesse, et peu d'estime pour son ennemie, se rangèrent de son côté ; quasi tous les hommes furent chez madame de Montbazon; et l'on compta jusqu'à quatorze princes qui la furent voir. Cette gloire, avec le plaisir de se venger de madame de Longueville, qui lui avait ôté un amant dont elle espérait un jour faire son mari, après la mort du sien qui était fort vieux, étaient des choses qui devaient donner assez de joie à une femme malicieuse, et qui ne voulait pas de réputation que celle de faire beaucoup d'éclat et d'avoir beaucoup d'amants. Mais tous les suppôts de sa vanité furent bientôt après contraints de se séparer d'elle, par la peur qu'ils eurent du jeune duc d'Enghien, qui, au bruit de la colère de madame la princesse, montra de vouloir porter les intérêts de madame de Longueville, sa sœur, avec beaucoup de chaleur. Cela les fit tous écarter fort aisément; car lui seul valait bien tous les quatorze ensemble. Parmi ce nombre, il faut en excepter, du côté de l'estime, M. de Nemours, qui venait d'épouser Mademoiselle de Vendôme, qui était un aimable prince et d'un grand mérite ; et respecter aussi la valeur extrême du duc de Guise, qui avait cette qualité en un éminent degré, quoique d'ailleurs on pût accuser sa conduite pour n'être pas toujours rêglée par la prudence et la raison.

En fait - privilège et pouvoir de l'équivoque -, l'usage des passions par la duchesse, qui lui assurait le soutien des courtisans, vient finalement la prendre au piège ; après la réaction de la princesse, ce sont ses appuis qui la trahissent, guidés par une nouvelle passion, la peur, que souligne ironiquement le féminisme de la mémorialiste. C'est aussi la passion qui va servir la princesse auprès de la reine, en même temps que les motifs politiques encadrés dans le récit par l'amour, puis les sentiments et la pitié de la reine :

La Reine, qui avait toujours aimé madame la princesse, se trouva fort disposée à la favoriser : elle était mère du duc d'Enghien, qui venait de gagner une bataille et qui se faisait déjà craindre : il fallait l'épargner tout à fait, de peur que le repos de la régence n'en fût troublé. Ces considérations devaient l'emporter sur tout le reste: la chose de soi l'obligeait aussi, et le droit des gens voulait qu'elle défendît la gloire de madame de Longueville, qui outre sa naissance avait de belles qualités, dont la réputation n'était point encore attaquée, et qui était fort aimable de sa personne. 
Cette princesse était grosse : elle était allée à La Barre, maison auprès de Paris qu'elle avait choisie pour aller passer les premiers chagrins de son aventure et pour s'y reposer. La Reine la fut voir pour la consoler et lui promettre sa protection. Après les premiers discours de civilité, madame la princesse la mena dans un cabinet où la mère et la fille se jetèrent à ses pieds, et lui demandèrent justice de l'outrage que madame de Montbazon leur avait fait. Ce fut avec tant de sentiments et tant de larmes, que la Reine, m'ayant fait l'honneur à son retour de La Barre de me conter ces particularités, me dit que ces princesses lui avaient fait pitié, et qu'elle leur avait promis qu'elles seraient entièrement satisfaites. Cela se fit en effet avec toute la cérémonie requise, et de manières qu'elles en furent contentes.

Ainsi la reine règle le problème, et Madame de Montbazon accepte la contre univoque (la rééquivocation) fixée par la reine. Mais la cérémonie est elliptique; les Mémoires de Mademoiselle de Montpensier, alors, sont précieux, parce qu'ils nous permettent de mieux comprendre comment s'opère cette dernière publication.

Le neutre faisait apparaître la laïcité de la fortune, la providence déchue ; l'équivoque fait apparaître l'indifférence des passions, leur profonde réversibilité.

On sait maintenant que l'équivoque se fixe en univoque par intervention du pouvoir ; on sait aussi que l'équivoque est travaillée par (et travaille) les passions du public (et c'est sans doute pourquoi celui-ci ne peut fixer l'équivoque en univoque, parce qu'il est changeant). S'affrontent un principe d'équivocité (les passions ici) et un principe d'univocité (ici, l'autorité royale). Mais par quelle procédure exactement le second s'impose-t-il au premier?

\section{Le récit de la Grande Mademoiselle : équivoque et duplicité}

31 L'analyse menée par Mademoiselle de Montpensier est moins romanesque ; Mademoiselle produit les pièces du dossier, et n'accorde pas grande place aux circonstances de la découverte.

[...] Madame de Montbazon trouva un soir chez elle deux billets d'une dame à un cavalier; elle dit aussitôt qu'ils étaient de madame de Longueville, et que Coligni, qui l'était venu voir ce jour-là, les avait laissés tomber de sa poche. Il faut remarquer, dans cette histoire, que l'opinion médisante de la cour était que M. de Longueville aimait madame de Montbazon depuis longtemps; qu'il était bien avec elle, et que madame la Princesse lui avait défendu de la voir depuis son mariage. Avant que de dire quelle suite eut la pièce que madame de Montbazon prétendait faire à madame de Longueville, je veux mettre ici une copie des billets qu'on dit qu'elle avait trouvés, puisque j'en ai une très fidèle de fort bon lieu : elle m'a été donnée avec le titre.

Copie des lettres supposées qui furent trouvées chez madame de Montbazon. J'aurais beaucoup plus de regret du changement de votre conduite, si je croyais moins mériter la continuation de votre affection. Je vous avoue que tant que je l'ai crue véritable et violente, la mienne vous a donné tous les avantages que vous pouviez souhaiter. Maintenant n'espérez pas autre chose de moi que l'estime que je dois à votre discrétion. J'ai trop de gloire pour partager la passion que vous m'avez si souvent jurée, et je ne veux plus vous donner d'autre punition de votre négligence à me voir que celle de vous en priver tout à fait; je vous prie de ne plus venir chez moi, parce que je n'ai plus le pouvoir de vous le commander.

En voilà une; et voici en quels termes était l'autre :

De quoi vous avisez-vous après un si long silence? Ne savez-vous pas bien que la même gloire qui m'a rendue sensible à votre affection passée me défend de souffrir les fausses apparences de sa continuation? Vous dites que mes soupçons et mes inégalités vous rendent 
la plus malheureuse personne du monde. Je vous assure que je n'en crois rien, bien que je ne puisse nier que vous ne m'ayez parfaitement aimée, comme vous devez avouer que mon estime vous a dignement récompensé. En cela nous nous sommes rendus justice, et je ne veux pas avoir dans la suite moins de bonté, si votre conduite répond à mes interventions. Vous les trouveriez moins déraisonnables, si vous aviez plus de passion; et les difficultés de me voir ne feraient que l'augmenter au lieu de le diminuer. Je souffre pour n'aimer pas assez, et vous pour aimer trop. Si je vous dois croire, changeons d'humeur; je trouverai du repos à faire mon devoir, et vous devez y manquer pour vous mettre en liberté. Je n'aperçois pas que j'oublie la façon dont vous avez passé avec moi l'hiver, et que je vous parle aussi franchement que j'ai fait autrefois. J'espère que vous en userez aussi bien, et que je n'aurai point de regret d'être vaincue dans la résolution que j'avais faite de n'y plus retourner. Je garderai le logis trois ou quatre jours de suite, et l'on ne m'y verra que le soir ; vous en savez la raison.

Madame de Montbazon, avec ces deux lettres, débita cette circonstance à tant de personnes et avec tant de railleries qu'elle fut bientôt divulguée. Sitôt que madame la princesse en eut connaissance, son humeur haute et fière la fit éclater avec chaleur contre madame de Montbazon; chacun attribua la calomnie que celle-ci avait répandue à la haine et à la jalousie qu'elle avait contre madame de Longueville. Les amis de Madame la princesse allèrent lui offrir leurs services; la cour se partagea dans cette occasion; tous les importants prirent le partie de madame de Montbazon, et la reine ne manqua pas de prendre l'autre. Ce qui le fortifia encore de la plus grande partie de la cour, était que M. le duc d'Enghien, à présent $\mathrm{M}$. le Prince, venait de rendre un service si considérable à l'État par le gain de la bataille de Rocroy, qu'on ne lui en pouvait assez témoigner de gré. La gloire de ce prince, la réputation avec laquelle il revenait de la campagne, rendirent madame sa mère plus fière qu'à l'ordinaire ; et lorsqu'on vint à parler d'accommodement, elle voulut que madame de Montbazon lui fit satisfaction.

Pour la mémorialiste, Madame de Montbazon ne se livre à aucune procédure éditoriale, elle décide arbitrairement de l'auteur. D'emblée, Mademoiselle de Montpensier identifie les billets comme des lettres supposées. Madame de Montbazon accusée directement, elle est fautive, et son erreur est l'excès de railleries. Le récit de la grande Mademoiselle est donc un croisement de celui de La Rochefoucauld (avec la reprise du motif de la supposition) et de celui de Madame de Motteville (avec la reprise du motif des passions). Mais le partage de la cour est plus politique que chez Motteville; ne s'opposent plus les hommes et les femmes, mais les importants et la Reine (qui s'appuie sur Enghien) : on a donc contre les premiers l'union des deux sources d'autorité (la lignée et la force militaire). C'est dans sa conclusion que le récit de la Grande Mademoiselle devient plus original par les détails qu'il donne. C'est bien l'autorité royale qui doit venir contre univoquer les lettres (les rééquivoquer).

L'affaire fut longtemps en négociation, parce que cette dernière [Mme de Montbazon] ne voulait pas se soumettre; la reine interposa son autorité ; elle s'y résolut. Le jour qui fut choisi pour cette soumission, madame la Princesse assembla chez elle, où madame de Montbazon devait venir, tous ses amis et amies; de sorte qu'il se trouva une excessive quantité de monde à l'hôtel de Condé.

Le clan Condé veut montrer sa puissance; la plus grande publicité va venir entériner la réunivocation de la lettre, au point de s'adjoindre toute l'aide qu'il peut trouver, jusqu'à la narratrice, entraînée à son corps défendant.

Monsieur y était, et je ne pus à mon égard me défendre d'y aller, bien qu'alors je n'eusse pas d'amitié pour madame la Princesse ni pour pas un de sa famille; néanmoins je ne pouvais avec bienséance dans cette occasion prendre un parti contraire au sien, et c'était là un de ces devoirs de parenté dont l'on ne se peut défendre. 
Mais, face à cette puissance, Madame de Montbazon va elle aussi faire une sorte de démonstration de force :

Madame de Montbazon, qui était fort parée, entra dans la chambre de madame la Princesse avec beaucoup de fierté ; et lorsqu'elle fut près d'elle, elle lut dans un papier qui était attaché à son éventail les excuses qu'on lui avait prescrit de dire, qui étaient en ces termes: Madame, je viens ici pour vous protester que je suis très innocente de la méchanceté dont on m'a voulu accuser. Il n'y a aucune personne $d$ 'honneur qui puisse dire une calomnie pareille. Si j'avais fait une faute de cette nature, j'aurais subi les peines que la reine m'aurait imposées; je ne me serais jamais montrée dans le monde, et vous en aurait demandé pardon. Je vous supplie de croire que je ne manquerai jamais au respect que je vous dois, et à l'opinion que j'ai de la vertu et du mérite de madame de Longueville.

Réponse de madame la princesse à madame la duchesse de Montbazon Madame, je crois très volontiers l'assurance que vous me donnez de n'avoir nulle part à la méchanceté que l'on a publiée : je défère trop au commandement que la reine m'en a fait.

Rien de plus équivoque, donc, que la cérémonie même destinée à mettre fin à l'équivoque première. Certes, la duchesse dément ses propos calomnieux ; mais elle le fait par le biais d'une dénégation; et (c'est lié) l'argument invoqué n'est pas celui de la fausseté du propos tenu, mais celui de l'autorité royale : reconnaissance ironique de la primauté du politique dans l'exégèse. Autrement dit, tout en regrettant ce qu'elle a dit, Madame de Montbazon ne remet pas en cause la véridicité du propos qu'elle a tenu: elle affirme seulement qu'elle n'avait pas à le tenir. La duchesse, comme aussi la princesse, affirment qu'elles ont bien compris que la seule source d'univocation possible est la reine; et le public n'est là que pour enregistrer cet édit royal. Mademoiselle, du reste, ne l'ignore pas, et souligne l'équivocité du propos en rapportant le ton avec lequel il est tenu.

Quand on fait de ces actions, il n'est pas ordinaire ni facile de les faire de bonne grâce, et le ton de celui qui s'excuse montre bien que le cœur ne se repent point de la faute qu'il a commise. Aussi ce que madame de Montbazon dit ne fut pas mieux reçu qu'elle le prononça; madame la princesse lui fit un discours plus court que le sien, quoiqu'il le fût assez, d'un air peu radouci, et sans rien quitter de cette majesté dont elle savait si bien accompagner tout ce qu'elle faisait. Cela n'était qu'une apparence de raccommodement; aussi la réconciliation ne dura pas longtemps, comme on le verra ci-après.

L'équivoque n'a pas de fin propre: la chute du parti des Importants, seule, mettra un terme à l'affaire. L'équivoque perdure jusque dans la cérémonie qui doit y mettre fin : c'est qu'aussi il n'est pas de besoin de plus que d'une manifestation d'un dessein univoque, quel que soit l'équivoque des circonstances. Le principe d'autorité l'emporte sur le principe passionnel, non qu'il soit plus vrai, plus simple, ou d'une nature autre: c'est seulement qu'il est plus fort, et, peut-être, qu'il entretient avec l'équivoque un lien plus intime. Le neutre faisait apparaître la laïcité de la fortune, la providence déchue; l'équivoque fait apparaître le conventionnel nu du politique, et donc son caractère irrémédiable, interminable.

\section{Un récit anonyme : équivoque et romanesque}

De la « pièce » décrite dans ces Mémoires au roman, il y a bien des différences. Mais le goût d'un certain romanesque est parfois commun; c'est en tout cas ce qui anime le récit anonyme publié par Adolphe Chéruel à la suite des Mémoires de la grande Mademoiselle. C'est que l'équivoque, ici, est bien figuré comme un moyen du pouvoir : 
Anne de Bourbon, duchesse de Longueville, était alors une des plus agréables personnes du monde, tant par les charmes de son esprit que par ceux de sa beauté. Coligny, fils aîné du maréchal de Châtillon, l'aimait passionnément, et l'on dit qu'il en était aimé [...] on dit qu'il se servit d'un moyen assez fin et fort extraordinaire, pour lui découvrir sa passion. Le roman de Polexandre était alors fort à la mode [...] Le duc d'Enghien le lisait à toute heure et y remarqua une lettre tendre et passionnée, qu'il montra à Coligny, qu'il estimait infiniment. Celui-ci ne manqua pas de profiter d'une occasion si favorable, et proposa au duc d'Enghien d'en faire une copie et de la mettre dans la poche de la duchesse. Il ne se passait presque point de jour qu'il n'y eût à l'hôtel de Condé quelque espèce de fête, et l'on y dansait presque tous les soirs. La proposition de Coligny fut acceptée. [Il copia la lettre] et la donna au duc d'Enghien. Ce jour-là, tout le monde était paré, et la duchesse brillait de mille rayons. Le bal commença de bonne heure, et le duc ayant pris la main de sa sœur, badinant avec elle, exécuta aisément son dessein. Je n'en sais pas davantage; mais il y a apparence que la lettre fut lue, et que la duchesse ne s'en plaignit pas. Quoique cette digression soit un peu longue, j'ai cru qu'elle ne gâterait rien à l'histoire et qu'elle ne serait pas inutile à mon sujet.

Le motif de la lettre flottante est d'emblée lié à l'intrigue romanesque et galante. Car la lettre perdue n'est pas celle dont il est ici question, mais dont pourtant il est ici fait digression ; contrairement à ce que prétend le narrateur, l'anecdote est rien moins que nécessaire au récit, d'autant qu'elle permet de mettre en place, face à une situation particulièrement équivoque, un récit non moins équivoque : avant le récit d'une lettre perdue, les précisions données sur une autre lettre ne peuvent que créer l'ambiguïté - à tout le moins la suspicion - dans l'esprit du lecteur. Les personnages ainsi présentés comme des héros de roman, viennent alors se préciser la «cause » de la haine, économique, c'est-à-dire privée.

Il est vrai que ce n'était pas sans cause, parce que, outre la rivalité ordinaire entre des personnes galantes et d'un tel mérite, le mariage de la duchesse de Longueville avait privé la duchesse de Montbazon de vingt mille écus de pension bien réglée, que le duc lui donnait. Cependant elles se rendaient d'assez fréquentes visites, mais tout en se picotant. Enfin, la duchesse de Longueville étant allée chez la duchesse de Montbazon, celle-ci courut au-devant d'elle, et en la saluant, elle ramassa un billet qui était à terre, sans que l'autre s'en aperçût. La visite ne fut pas longue ; mais elle ne laissa pas de le paraître à la duchesse de Montbazon, qui brûlait d'impatience de voir ce billet. L'assemblée était belle et nombreuse ; les ducs de Guise et de Beaufort en étaient. Le billet fut lu en pleine assemblé, et la charité publia qu'il était tombé de la poche de la duchesse de Longueville, et que Coligny en était l'auteur ; d'autres l'attribuaient à Lesdiguières. Mais le duc de Guise me fit l'honneur de me dire qu'il venait de madame de Fouquerolles, à qui Maulevrier l'avait écrit.

Le détail de la scène souligne l'œuvre de la volonté. L'univocité à venir est déjà dans l'équivoque de cette inclination qui est soumission, et moyen de se procurer le piège. Le geste équivoque conduit à l'objet équivoque, comme la digression première avait conduit le récit dans un dispositif équivoque. Mais la haine de la duchesse, sur laquelle le mémorialiste insiste plus que les autres, est le moteur. C'est là encore la passion qui vient fabriquer l'équivoque, puis l'univoque (parce que l'équivoque n'est pas une arme, mais le moyen d'en fabriquer une).

La princesse de Condé[...] ne manqua [...] pas le lendemain d'en porter ses plaintes à la reine mère et de lui demander justice de l'impudence et de la calomnie de la duchesse de Montbazon. La reine se trouva fort embarrassée, lui fit des réponses ambiguës; mais se voyant pressée et considérant l'importance de cette affaire, après en avoir conféré avec le cardinal de Mazarin, elle prit enfin son parti, c'est-àdire qu'elle ordonna à la duchesse de Montbazon d'aller, dès le lendemain, à l'hôtel 
de Condé faire une réparation publique à la duchesse de Longueville, et la prier de lui vouloir pardonner. Cet arrêt était sévère ; cependant il fallut l'exécuter.

38 À nouveau, la demande de justice passe par l'exigence d'une contre univocation royale. Il s'agit donc non de retourner à l'équivoque - les réponses ambiguës de la reine -, mais bien de contre univoquer l'objet. Le public, là encore, doit entériner ce geste que seule l'intervention royale permet. La fixation de l'objet équivoque en objet contre univoqué dépend bien de l'intervention royale. Mais une nouvelle équivoque se produit au cours de la réparation : on assiste presque à une inversion des rôles.

La duchesse de Montbazon [...] se rendit à l'hôtel de Condé sur les quatre heures du soir. Jamais elle ne parut si belle, et, à voir son air libre et dégagé, on eût cru qu'elle venait plutôt pardonner que s'excuser. [...] Alors il se fit un silence de chartreux, et la duchesse de Montbazon prenant la parole, fit un désaveu général de tout ce qu'on avait publié sous son nom ; elle dit de fort bonne grâce qu'elle venait pour obéir au commandement de la reine ; qu'elle avait trop de respect pour le sang royal et pour la duchesse de Longueville pour manquer jamais à son devoir. La princesse de Condé répondit qu'elle avait toujours bien cru ce qu'elle disait; qu'elle était très aise de l'apprendre de sa bouche, et qu'elle en demeurait persuadée. Ainsi finit la comédie, et la compagnie se sépara. [Suit le récit du duel entre Guise et Coligny, lié à la rivalité des duchesses] On peut dire enfin que voilà l'origine de tous les désordres et de tous les troubles dont la France a été agitée depuis si longtemps. Comme chez la Grande Mademoiselle, la mention de l'autorité royale par Madame de Montbazon est équivoque. Elle renverse l'excuse et ainsi l'équivoque : ce n'est pas en raison de la réalité des actions que la duchesse dément, mais par respect pour l'autorité royale. Cette dissociation manifeste du discours et de la pensée fait bien de cette scène une "comédie", et l'équivoque se rouvre, comme si elle ne pouvait pas se fermer dans un geste univoque. C'est que l'anecdote occupe pour l'auteur une place centrale dans le déclenchement de la Fronde : cette fin n'est pas une fin. La part de romanesque dans ce texte permet alors de mieux comprendre le succès de l'équivoque au $\mathrm{XVII}^{\mathrm{e}}$ siècle: la force narrative de l'équivoque consiste précisément à ne pas fermer le récit, mais à l'ouvrir à des possibles différents, dans lesquels l'Histoire peut se glisser; si celle-ci devient "neutre ", c'est aussi qu'elle devient, pour ces perdants que sont la plupart des mémorialistes, illisible; l'équivoque est le signe de cette illisibilité, ce qui donne à voir l'indéterminé des choses dans son inquiétante - et fascinante - obscurité.

39 À suivre le récit de trois mémorialistes, l'exégèse comme procédure de vérité est d'abord affaire de pouvoirs : pouvoir insuffisant de Madame de Montbazon qui échoue à imposer sa vengeance, mais qui se sort de l'affaire par une excuse équivoque; pouvoir du clan Condé récemment renforcé par la victoire militaire de l'aîné ; pouvoir souverain de la reine qui fixe les règles d'univocation; pouvoir enfin, jamais nommé sinon par le soupçon, de Mazarin qui renverse les fortunes. D'où le privilège d'un objet équivoque, visage du neutre : il manifeste l'indifférence de la vérité, la raison politique de l'exégèse, et par là révèle le conventionnel nu du politique. Aussi bien s'explique la fascination qu'a pu exercer cette anecdote sur les contemporains : elle dit le caractère paradoxal d'une épistémé où le vrai ne coïncide pas nécessairement avec les faits, où l'usage importe plus que l'objet qu'il met en jeu, où l'équivoque est plus vrai que les différents univoques que l'on peut prétendre lui substituer. Ce qui se donne dans l'équivoque, c'est à la fois le spectacle des pouvoirs, et des pouvoirs qui s'affrontent et se jaugent, et la possibilité d'une représentation de l'ambiguité des pouvoirs, de leur toujours menaçante réversibilité. À travers cette affaire de lettre perdue, qui mélange rivalité galante et intrigue politique, c'est aussi le succès des formes fragmentaires au XVII ${ }^{\mathrm{e}}$ siècle qui s'éclairent, objets sans contexte, et à ce titre particulièrement équivoques : les maximes 
de La Rochefoucauld, alternativement lues comme augustiniennes et comme libertines, en seraient un autre exemple. Mais, avant que de trancher la question de l'interprétation de ces objets duplices (c'est, semblerait-il, d'abord affaire de pouvoirs), il faut rendre compte de cette duplicité elle-même, et de ce qu'elle dit du statut particulier de la vérité à l'âge classique.

\section{NOTES}

1. Louis Marin, «Bagatelles pour meurtres ", dans Le Récit est un piège, Paris, éditions de Minuit, 1978, p. 65.

2. Voir notamment Jean Lafond, éd., Les Formes brèves de la prose et le discours discontinu aux XVI et XVII ${ }^{e}$ siècles, Paris, Vrin, 1978 ; Antoine Compagnon, La Seconde Main, ou le travail de la citation, Paris, Seuil, 1979 ; Lucien Dallenbach et Christian L. Hart, Fragments et totalité, Francfort-sur-Main, Suhrkamp, 1982; Marc Escola, "Ceci n'est pas un livre. Prolégomènes à une rhétorique du discontinu ", Dix-septième siècle, $\mathrm{n}^{\circ} 182$, janv.-mars 1994, p. 71-82; Georges May, La Perruque de Dom Juan, ou du bon usage des énigmes dans la littérature de l'âge classique, Paris, Klincksieck, 1995 ; Françoise Jaouen, De l'art de plaire en petits morceaux. Pascal, La Rochefoucauld, La Bruyère, Vincennes, Presses Universitaires de Vincennes, 1996; Bernard Roukhomovsky et Louis Van Delft, « Les formes brèves et le fragment ", Dix-septième siècle, $\mathrm{n}^{\circ}$ 202, janv.-mars 1999, p. 157-168.

3. La Rochefoucauld, Mémoires, dans Maximes, Mémoires, Euvres diverses, Jacques Truchet, Marc Escola, Alain Brunn, éd., La Pochothèque/Classiques Garnier, 2001, p. 908 sq.; Mémoires de Madame de Motteville, édition de Francis-Marie Riaux, Charpentier, Paris, s. d. ; p. 135 sq., chapitre VII ; Mémoires de Mademoiselle de Montpensier, éd. Adolphe Chéruel, Paris, Charpentier, 1858 ; t. 1, p. 76 sq. ; auteur anonyme, cité par Adophe Chéruel, op. cit., p. 386. On a omis les récits trop rapides de La Châtre, d'Ormesson, de Campion et de Retz.

4. Michel Foucault, «Qu'est-ce qu'un auteur? », Bulletin de la société française de philosophie, $\mathrm{n}^{\circ} 63$, 1969. Voir notamment l'analyse de la méthode de Jérôme : «si, parmi plusieurs livres attribués à un auteur, l'un est inférieur aux autres, il faut le retirer de la liste de ses œuvres (l'auteur est alors défini comme un certain niveau constant de valeur); de même, si certains textes sont en contradiction de doctrine avec les autres œuvres d'un auteur (l'auteur est alors défini comme un certain champ de cohérence conceptuelle ou théorique) ; il faut également exclure les œuvres qui sont écrites dans un style différent, avec des mots et des tournures qu'on ne rencontre pas d'ordinaire sous la plume de l'écrivain (c'est l'auteur comme unité stylistique); enfin on doit considérer comme interpolés les textes qui se rapportent à des événements ou qui citent des personnages postérieurs à la mort de l'auteur (l'auteur est alors moment historique défini et point de rencontre d'un certain nombre d'événements). »

5. Il s'agit à la fois de rééquivoquer la lettre (rouvrir le champ des auteurs possibles), tout en la contre univocant (un auteur - Madame de Longueville - est exclue de ce champ).

6. Molière, Le Misanthrope, II, 4. Alceste s'adresse au public de Célimène, ces petits marquis qui s'étonnent de le voir les critiquer et d'épargner sa belle : « Non, morbleu ! c'est à vous ; et vos ris complaisants/ Tirent de son esprit tous ces traits médisants. »

7. On pense bien sûr ici autant à Machiavel (Le Prince, chapitre XVIII) qu'à certains motifs pascaliens, qui nouent le politique et le divertissement (Pensées, Sellier 457 : « quand [Platon et Aristote] se sont divertis à faire leurs Lois et leurs Politiques, ils l'ont fait en se jouant »). 